\title{
Real Structure of Benzene Molecule and the Thickness of Graphene
}

\section{Dulun Wang ( $\nabla$ gzwdl@163.com )}

Guangdong Pharmaceutical University

\section{Research Article}

Keywords: benzene, molecular structure, half-valent bond, hydrogenation heat, ultraviolet spectrum, graphene thickness

Posted Date: December 13th, 2021

DOI: https://doi.org/10.21203/rs.3.rs-1157910/v1

License: (c) (i) This work is licensed under a Creative Commons Attribution 4.0 International License. Read Full License 


\section{Abstract}

The problem of benzene molecular structure has not been solved for more than 100 years. This research proposes a new concept of covalent bond based on the existing theory: each electron shared by multiple atom nuclei corresponds to a half-valent bond. The half-valent bond can be formed between the spacer carbon atoms of the benzene ring. In this way, a new theory was established. Quantum mechanical calculations can quantitatively explain experimental results, such as the hydrogenation heat and ultraviolet spectrum of benzene. Using the dotted line to indicate the half-valent bond, benzene molecular structural forms and chemical reaction formulas as will as its dozens of homologues and derivatives are designed easily. The method not only has a wide range of adaptability, but can also record the chemical reaction process. If several stacked benzene rings can form a benzene tube under the guidance of the new theory, calculated thickness of the three-layer benzene tube is very close to the thickness of graphene. Therefore, referring to other characteristics of graphene, it is considered to be more like a threelayer structure.

\section{Introduction}

Since F.A.Kekulé proposed the benzene molecular plane structure of cyclohexatriene, which was inconsistent with the experimental results. Then some people have proposed also some related structures, which were all unsuccessful. In particular, the experimental results of the hydrogenation heat and the ultraviolet spectrum of benzene that cannot be quantitatively explained. Later it became the Mystery of a century ${ }^{1,2,3}$.

\section{New theory of the covalent bond}

Solving the problem of benzene molecular structure should follow the valence bond theory of chemical bond $^{4,5}$. The nature of chemical bond is that nuclei share electrons. In the existing valence bond theory, nuclei share a pair of opposite spin electrons to form one valence bond ${ }^{6}$. This definition is not suitable for the reality of benzene and some other molecules. Moreover, the theory has never been proven that the shared electrons must be paired and the spins must be reversed ${ }^{7,8}$. Therefore, here is an attempt to discard the above two insufficient and nonessential conditions. In the new theory, it is defined as each electron shared by nuclei should correspond to a half-valent bond.

\section{New planar structure of benzene molecule}

According to the existing molecular orbital theory, the $2 s$ and $2 p$ orbital of every carbon atom in the benzene ring may be hybridized into three $s p^{2}$ orbital and one alone $p_{z}$ orbital. $12 s p^{2}$ orbital of the 6 carbon atoms form $6 \sigma$ bonds and are connected into a regular hexagonal ring. The other $6 \sigma$ bonds are connected outside the ring to the hydrogen atom. The remaining 6 unhybridized $p_{\mathrm{z}}$ orbital are linearly combined into 6 molecular orbital. Among them, there are 3 bonding orbital and 3 anti-bonding orbital. Their $6 p$ electrons are all on the bonding orbital, and each orbital can accommodate 2 electrons with 
opposite spin directions. The 3 anti-bonding orbital are unoccupied and vacant. The molecular structural energy is low, so the structure is stable ${ }^{9,10} .6$ carbon nuclei of the benzene ring share $6 p$ electrons, forming 6 half-valent $\pi$ bonds and connected into a large half-valent $\pi$ bond ring. It is the ground state of the benzene molecule.

When getting enough energy outside the system, one electron in each bonding orbital jump to the antibonding orbital. 3 anti-bonding orbital are full occupied and all electrons become the same $\operatorname{spin}^{11}$. The active electrons increase the mutual repulsion between adjacent $p_{\mathrm{z}}$ orbital electrons. The steric hindrance effect ${ }^{9}$ causes one of the $p_{z}$ orbital to turn to the opposite location phase of the benzene ring plane. Since then, each both positive or negative location phases have three interval carbon atom $p_{\mathrm{z}}$ orbital, and forming a new neighboring relationship of triangular rings. In each phase, there are three interval carbon nuclei sharing three $\pi$ electrons, forming three half-valent $\pi$ bonds and their bond rings. This is the excited state of the benzene molecule. Only molecules in an excited state containing active electrons can participate in chemical reactions.

Such a valence bond theory follows the tetravalent of carbon and satisfies requirement of the saturation of the covalent bond ${ }^{4}$, and make the molecular structure still stable.This series of concepts formed a new theory of the planar structure of the benzene molecule.

\section{Benzene tube hypothesis}

Assuming that in the excited state of benzene, when the flipped ring plane of one molecule overlaps with another molecule, the two benzene rings are parallel and mirror-symmetrical. They correspond to the three $p_{\mathrm{z}}$ orbitals of the spaced carbon atoms, butt to form three $\sigma$ bonds, and connect to form a two-layer benzene tube. The other three spaced $p_{z}$ orbitals of the flipped ring plane are connected to another molecule that is not flipped in the same way, and finally a three-layer benzene tube is formed. The top and bottom of the three-layer benzene tube are exactly the same as the top and bottom of the benzene molecule, and it will be as stable as benzene. However, since multiple vertical sigma bonds form a lattice, the physical properties will be like metals. After calculating the thickness of the three-layer benzene tube, it is very close to the thickness of graphene. The physical properties of graphene are also metal-like. Therefore, it is speculated that graphene is more like a three-layer structure.

\section{Method}

In order to verify the correctness of the new theory of benzene molecular structure, it is necessary to establish the electronic wave function of the benzene ring and its wave equation and solve the equation. Use relevant calculated values to quantitatively explain experimental results. In addition, to design a molecular structural formula that conforms to the new theory, the design method must not only have a wide range of adaptability to design any molecule, but also be able to record many details of its chemical reaction process. 


\section{Quantum mechanical calculations verify the reliability of the new structural theory}

A complex wave equation with multiple electrons, and it is almost impossible to solve $\mathrm{e}^{2,12}$ it. Only a simplified model can be used to approximate the solution.

In the benzene ring, $6 p$ electrons rotate around the carbon nucleus where they are located, and the movement of each electron has an arc in the same direction along the benzene ring. They will be connected in 6 arc to form a circle. The interaction of the corresponding 6 carbon nuclei with electrons is regarded as the positive charge of the center. In this way, their overall behavior is similar to an equivalent electron, which moves in a circle around the positive charge along the circumcircle of the benzene ring. The ground state and the excited state are the two energy levels of equivalent electrons. Thus, a simplified equivalent electronic model of benzene molecule was established. If the model can be transitioned to the hydrogen-like atom model ${ }^{7}$, the existing method ${ }^{12}$ can be used to those calculations. However, the kinetic energy of the equivalent electron is unknown. The only data that can be inferred is the work done by the electrons around the circumscribed circle of the benzene ring.

$$
W_{H}=2 \Pi f_{H} r_{H}
$$

(1)

The question is whether it is possible for $W_{H}$ to be seen as kinetic energy $E_{K}$. First look, the circumference of the circle is expressed by integral and the force is expressed by the derivative of speed.

$$
l=2 \pi r_{H}=\int_{0}^{t_{0}} u d t, f_{H}=m \frac{d u}{d t}
$$

So, (1) becomes

$$
W_{H}=2 \pi f_{H} r_{H}=f_{H} l=m \frac{d u}{d t} \int_{0}^{t_{0}} u d t=m \int_{0}^{u_{0}} u d u=\frac{1}{2} m u_{0}^{2}
$$

3

It seems that at the end point of the circle, $W_{H}$ and kinetic energy $E_{K}$ can be equal.

$$
W_{H}=E_{K}
$$

4

It can also be said that at this point, it is possible to change the equivalent electron model to a hydrogenlike atom model. Similarly, at this point, the kinetic energy $E_{K}$ and the potential energy $V_{H}$ of the electrons outside the hydrogen nucleus are also equal.

In the same way, the kinetic energy $E_{K}$ and the potential energy $V_{H}$ of the electrons outside the hydrogen nucleus are also equal. 


$$
W_{H}=E_{K}=V_{H}=\frac{e^{2}}{4 \pi \varepsilon_{0} r_{H}}
$$

5

$2 \Pi f_{H} r_{H}=\frac{\mathrm{e}^{2}}{4 \Pi \varepsilon_{0} r_{H}}, f_{H}=\frac{e^{2}}{8 \Pi^{2} \varepsilon_{0} r_{H}^{2}}(6)$

In (5), $e$ is the electronic charge, and $\varepsilon_{0}$ is the vacuum permittivity. The force of the equivalent electron should be the same as the general form of the force of the hydrogen atom, and when it is equal to the force of the extranuclear electron of hydrogen, it must be

$$
\begin{gathered}
f_{B}=\frac{e^{2}}{4 \Pi \varepsilon_{0} r_{B}^{2}}, \\
f_{B}=f_{H^{\prime}} \\
\frac{e^{2}}{4 \Pi \varepsilon_{0} r_{B}^{2}}=\frac{e^{2}}{8 \Pi^{2} \varepsilon_{0} r_{H}^{2}}
\end{gathered}
$$

7

$\ln (7)$, there are

$$
r_{B}^{2}=2 \Pi r_{H}^{2}
$$

8

In (8), r only represents feasible in the simplification of the equivalent electron-like hydrogen atom model. However, there is an error between it and the actual situation. Therefore, let $g$ here, as a real coefficient that has nothing to do with $r$, to represent the error. get

$$
r_{B}^{2}=2 \pi g r_{H}^{2}
$$

9

This clearly shows that the difference in the expression of the two forces is mainly reflected in the radius $r$. If the different $r$ at both ends of formula (9) can be regarded as a coordinate transformation, this transformation can make the equivalent electron model transition to the hydrogen-like atom model ${ }^{13}$.

With reference to (7), (8) and (9), the potential energy of the equivalent electron can also be written more in the general form ${ }^{14}$ :

$$
\begin{aligned}
V_{B}=\int \frac{e^{2}}{8 \Pi^{2} g \varepsilon_{0} r^{2}} d r= & -\frac{e^{2}}{16 \Pi^{2} g \varepsilon_{0} r}=-\frac{e^{2}}{8 \Pi^{2} b \varepsilon_{0} r} \\
b & =2 g
\end{aligned}
$$


In (10), $V_{B}$ is the equivalent electronic potential energy of the benzene ring.So, as the equivalent electronic coordinate transformation of the benzene ring, refer to (9) and (10), which can be written as a general transformation form:

$$
r_{B}^{2}=\Pi b r^{2}=\Pi b\left(x^{2}+y^{2}+z^{2}\right)
$$

$$
r_{B}=r(x, y, z) \sqrt{\Pi b}
$$

11

Based on the above-mentioned equivalent electrons, it makes a reciprocating circular motion along the benzene ring, and only at the end of the circle meets the conditions of the above model. According to these conditions, imitating Schrödinger, the equivalent electronic wave function is established ${ }^{7}$. Due to the limitation of (3), the wave function period can only be set to $2 n \pi$. From (4), (10), (11), Planck formula and De Broglie formula ${ }^{7}$, get

$$
\begin{aligned}
& \Psi=e^{-i 2 n \pi\left(v t-\frac{r \sqrt{\pi b}}{i}\right)}=e^{-i \frac{2 n \pi}{h} E t} \psi, \\
& \psi=e^{i \frac{2 n \pi}{h} p r \sqrt{\pi b}} \\
& E_{K}=h v=\frac{1}{2} m u^{2}, \lambda=\frac{h}{p}, p=m u=\sqrt{2 m E}, u=\lambda v, E_{K}=E-V
\end{aligned}
$$

In (12), $n$ is a natural number, $\lambda$ is the wavelength, $v$ is the frequency, $r$ is the radius of the benzene $r i n g, h$ is the Planck constant, $p$ is the electron momentum, $m$ is the static mass of the electron, $u$ is the electron velocity, $E_{K}$ is the kinetic energy, $E$ is the total energy, and $V$ is the potential energy. With reference to (10), (12) and Schrodinger equation ${ }^{7,12}$, the three-dimensional wave equation of the equivalent electron can be obtained:

$$
\frac{\partial^{2} \psi}{\partial x^{2}}+\frac{\partial^{2} \psi}{\partial y^{2}}+\frac{\partial^{2} \psi}{\partial z^{2}}=\frac{8 n^{2} \Pi^{3} b m}{h^{2}}(E-V) \psi
$$

13

Substitute (10) into (13) to get

$$
-\frac{h^{2}}{8 n^{2} \Pi^{3} b m}\left(\frac{\partial^{2} \psi}{\partial x^{2}}+\frac{\partial^{2} \psi}{\partial y^{2}}+\frac{\partial^{2} \psi}{\partial z^{2}}\right)-\frac{e^{2}}{8 \Pi^{2} b \varepsilon_{0} r} \psi=E \psi
$$


Equation (14) is converted to the spherical coordinate equation

$-\frac{h^{2}}{8 n^{2} \Pi^{3} b m}\left[\frac{1}{r^{2}} \frac{\partial \psi}{\partial r}\left(r^{2} \frac{\partial \psi}{\partial r}\right)+\frac{1}{r^{2} \sin \theta}\left(\sin \theta \frac{\partial}{\partial \theta}\right)+\frac{1}{r^{2} \sin ^{2} \theta} \frac{\partial^{2} \psi}{\partial \psi^{2}}\right]-\frac{e^{2}}{8 \Pi^{2} b \varepsilon_{0} r} \psi=E \psi$

In order to solve the energy eigenvalue $E$ in equation (15), the variational method will be used. Here, the Lagrangian undetermined coefficient method is applied ${ }^{13,14}$. The Hamiltonian ${ }^{13,14}$ is used in the algorithm. Therefore, the following conditions must be met:

$$
\begin{aligned}
& H \psi=E \psi, \delta\left(\int \psi^{*} H \psi d u-\eta \int \psi^{*} \psi d u d\right)=0 \\
& (H-\eta) \psi=0, \delta \eta(\psi) \equiv \delta\left(\frac{\int \psi * H \psi d u}{\int \psi * \psi d u}\right)=0
\end{aligned}
$$

In (16) and (17), $H$ is the Hamiltonian quantity, $E$ is the energy eigenvalue, and $\eta$ is the undetermined coefficient. Since only the $r$ part of (15) is involved, $H$ can be

$$
\begin{aligned}
H=-\frac{h^{2}}{8 n^{2} \Pi^{3} b m}\left[\frac{1}{r^{2}} \frac{\partial}{\partial r}\left(r^{2} \frac{\partial}{\partial r}\right)\right]-\frac{e^{2}}{8 \Pi^{2} b \varepsilon_{0} r} & \\
& =-\frac{h^{2}}{8 n^{2} \Pi^{3} b m}\left(\frac{\partial^{2}}{\partial r^{2}}+\frac{2}{r} \frac{\partial}{\partial r}\right)-\frac{e^{2}}{8 \Pi^{2} b \varepsilon_{0} r}
\end{aligned}
$$

18

Suppose: the variational function is $\psi=e^{-a r}$, and $a$ is a positive real number. Through (16), (17) and (18), the undetermined coefficient $\eta$ can be calculated by the variational method as follows:

$$
\begin{aligned}
& \eta=\frac{-4 \Pi \int_{0}^{\infty} e^{-a r}\left\{\left[\frac{h^{2}}{8 n^{2} \Pi^{3} b m}\left(\frac{\partial^{2}}{\partial r^{2}}+\frac{2}{r} \frac{\partial}{\partial r}\right)+\frac{e^{2}}{8 \Pi^{2} b \varepsilon_{0} r}\right] e^{-a r}\right\} r^{2} d r}{4 \Pi \int_{0}^{\infty} r^{2} e^{-2 a r} d r} \\
& =\frac{-\frac{h^{2} a^{2}}{8 n^{2} \Pi^{3} b m} \int_{0}^{\infty} r^{2} e^{-a r} d r+\left(\frac{h^{2} a}{8 n^{2} \Pi^{3} b m}-\frac{e^{2}}{8 \Pi^{2} b \varepsilon_{0}}\right) \int_{0}^{\infty} e^{-a r} r d r}{\int_{0}^{\infty} e^{-2 a r r^{2} d r}}
\end{aligned}
$$




$$
\eta=\frac{h^{2} a^{2}}{8 n^{2} \Pi^{3} b m}-\frac{e^{2} a}{8 \Pi^{2} b \varepsilon_{0}}
$$

19

by (16), (17) and (19)

$$
\frac{d \eta}{d a}=\frac{h^{2} a}{4 n^{2} \Pi^{3} b m}-\frac{e^{2}}{8 \Pi^{2} b \varepsilon_{0}}=0
$$

$$
a=\frac{n^{2} \Pi m e^{2}}{2 \varepsilon_{0} h^{2}}
$$

20

Substituting (20) into (19), the coefficient $\eta$ to be determined is

$$
\begin{gathered}
\eta=\frac{h^{2}}{8 n^{2} \Pi^{3} b m}\left(\frac{n^{2} \Pi m e^{2}}{2 \varepsilon_{0} h^{2}}\right)^{2}-\frac{e^{2}}{8 \Pi^{2} b \varepsilon_{0}}\left(\frac{n^{2} \Pi m e^{2}}{2 \varepsilon_{0} h^{2}}\right) \\
=-\frac{n^{2} m e^{4}}{32 \Pi b \varepsilon_{0}^{2} h^{2}}
\end{gathered}
$$

21

From (16), (17), (18), (19) and (21), calculated energy eigenvalue ${ }^{14}$ is

$$
E=-\frac{n^{2} m e^{4}}{32 \pi b \varepsilon_{0}^{2} h^{2}}
$$

22

1) Quantitatively explain the hydrogenation heat of benzene with the quantum mechanics calculation

In (22), $E$ is the energy eigenvalue of the equivalent electron. Turn it into an energy level formula is

$$
E_{n}=\frac{n^{2} E_{H}}{4 \Pi b},(n=0,1,2),
$$

23

$E_{H}=-\frac{m_{e} e^{4}}{8 \varepsilon_{0}^{2} h^{2}}=-13.606(\mathrm{eV})=313.755(\mathrm{kcal} / \mathrm{mol})$

In (23),(24), $E_{H}$ is the lowest energy level of extranuclear electrons of hydrogen atom ${ }^{7} . m$ is the static mass of electron, $m=9.1095 \times 10^{-31} \mathrm{~kg}$, basic charge $e=1.602 \times 10^{-19} \mathrm{C}$, the vacuum dielectric constant 
$\varepsilon_{0}=8.854 \times 10^{-12} \mathrm{~F} / \mathrm{m}$, Planck constant $h=6.626 \times 10^{-34} \mathrm{~J}$.S. $E_{n}$ is the energy level of the equivalent electron. $n$ is the energy level number. When $n=2$, the energy level is $E_{2}$, which is the lowest energy level. In the ground state. When $n=1$, the energy level is $E_{1}$ and it is in an excited state. Active electrons can participate in chemical reactions. The electrons further increase energy and escape beyond the control of the benzene ring. The benzene ring is destroyed and benzene is hydrogenated to form cyclohexane. If it is also regarded as an extreme state of energy level, it can only be $n=0$ and energy level $E_{0}=0$. These energy levels are as follows:

$E_{0}=0$

$$
E_{1}=\frac{E_{H}}{4 \Pi b}=-2.165 / 2 b(e V)
$$

25

$$
E_{2}=4 E_{1}=-8.658 / 2 b(e V)
$$

26

Their transition energies should are

$$
\Delta E_{1}=E_{0}-E_{1}=2.165 / 2 b(e V)
$$

27

$$
\Delta E_{2}=E_{1}-E_{2}=3 E_{1}=6.493 / 2 b(e V)
$$

28

Where $\Delta E_{2}$ is the transition energy of the ground state. $\Delta E_{1}$ is the transition energy of the excited state.The transition energy of the equivalent electron in the excited state was used to quantitative interpretation the experimental results of the hydrogenation heat of benzene $e^{2,6}$. In (27), the transition energy value $\Delta E_{7}=2.165 / 2 \mathrm{~b}(\mathrm{eV})$ of the excited state is equivalent to the hydrogenation heat released of benzene to cyclohexane. The experimental value of the hydrogenation heat of benzene is $2.160 \mathrm{eV}$ $(49.802 \mathrm{kcal} / \mathrm{mol})$. The error rate is only $0.231 \%(2 b=1.00231)$. It is close enough. The experimental result of the hydrogenation heat of benzene is quantitatively explained here.

If $2 b$ is ignored, the hydrogenation heat of benzene is a constant composed entirely of basic physical constants $^{12}$, denoted as $B_{H}$ 


$$
B_{H}=\Delta E_{1}=\frac{m e^{4}}{16 \pi \varepsilon_{0}^{2} h^{2}}
$$

$$
\begin{aligned}
& \Delta E_{2}=E_{1}-E_{2}=\frac{h c}{\lambda}=-\frac{m e^{4}}{16 \pi \varepsilon_{0}^{2} h^{2}}\left(n_{1}^{2}-n_{2}^{2}\right)=3 B_{H} \\
& \frac{3 m e^{4}}{16 \pi \varepsilon_{0}^{2} h^{3} c}=\frac{3}{2 \pi} R_{\infty}, R_{\infty}=\frac{2 \pi}{h c} B_{H} \\
& R_{\infty}=\frac{m e^{4}}{8 \varepsilon_{0}^{2} h^{3} c}=1.097 \times 10^{7}\left(\mathrm{~m}^{-1}\right)
\end{aligned}
$$

In (29) and (30), $c$ is the speed of light, $c=2.998 \times 10^{8} \mathrm{~m} / \mathrm{s}$. Since the ground state transition energy $\Delta E_{2}$ is related to the ultraviolet light energy absorbed by benzene, the Rydberg constant $R_{\infty}$ can also be measured by the ultraviolet spectroscopy.

\section{2)Quantitative interpretation of the UV spectrum of benzene with quantum mechanics calculation}

If the energy level of the equivalent electron is related to the energy level of the molecular orbital, here, through the simplified calculation of the Hückel molecular orbital theory 7,17 , the wave function and energy level of the molecular orbital of benzene are:

$$
\begin{aligned}
& \psi_{1}=\frac{1}{\sqrt{6}}\left(\phi_{1}+\phi_{2}+\phi_{3}+\phi_{4}+\phi_{5}+\phi_{6}\right), \quad \varepsilon_{1}=\alpha+2 \beta \\
& \psi_{2}=\frac{1}{\sqrt{12}}\left(2 \phi_{1}+\phi_{2}-\phi_{3}-2 \phi_{4}-\phi_{5}+\phi_{6}\right), \quad \varepsilon_{2}=\alpha+\beta \\
& \psi_{3}=\frac{1}{\sqrt{4}}\left(\phi_{2}+\phi_{3}-\phi_{5}-\phi_{6}\right), \quad \varepsilon_{3}=\alpha+\beta \\
& \psi_{4}=\frac{1}{\sqrt{4}}\left(\phi_{2}-\phi_{3}+\phi_{5}-\phi_{6}\right), \quad \varepsilon_{4}=\alpha-\beta \\
& \psi_{5}=\frac{1}{\sqrt{12}}\left(2 \phi_{1}-\phi_{2}-\phi_{3}+2 \phi_{4}-\phi_{5}-\phi_{6}\right), \quad \varepsilon_{5}=\alpha-\beta \\
& \psi_{6}=\frac{1}{\sqrt{6}}\left(\phi_{1}-\phi_{2}+\phi_{3}-\phi_{4}+\phi_{5}-\phi_{6}\right), \quad \varepsilon_{6}=\alpha-2 \beta
\end{aligned}
$$

According to the new theory of benzene molecular structure, referring to (25), (26), (31), the corresponding ground state and excited state energy levels are 


$$
\begin{aligned}
& E_{1}=\varepsilon_{1}+\varepsilon_{2}+\varepsilon_{3}+\varepsilon_{4}+\varepsilon_{5}+\varepsilon_{6}=6 \alpha=-2.165(\mathrm{eV}) \\
& E_{2}=2\left(\varepsilon_{1}+\varepsilon_{2}+\varepsilon_{3}\right)=6 \alpha+8 \beta=-8.660(\mathrm{eV}) \\
& \Delta E_{2}=E_{1}-E_{2}=6.493(\mathrm{eV})=-8 \beta
\end{aligned}
$$

from (27), (29), (32) get

$$
\alpha=-0.361(\mathrm{eV})=-\frac{m_{e} e^{4}}{6 \times 16 \pi^{2} \varepsilon_{0}^{2} h^{2}}(\mathrm{eV})
$$

from (29) (30) (34), get

$$
\beta=-0.812(\mathrm{eV})=-\frac{\left(1-2^{2}\right) m_{e} e^{4}}{-8 \times 16 \pi^{2} \varepsilon_{0}^{2} h^{2}}(\mathrm{eV})
$$

In (35), The Coulomb integral $a$ and the transition integral $\beta$ in the Hückel molecular orbital theory are constants composed of basic physical constants. The relationship is obtained between $a$ and $\beta$

$$
6 \alpha=\frac{3}{8} \beta=-\frac{m_{e} e^{4}}{16 \pi^{2} \varepsilon_{0}^{2} h^{2}}(e V), \quad \alpha=\frac{\beta}{16}
$$

The UV spectrum ${ }^{16,17}$ of benzene shows that it has a strong single peak at $184 \mathrm{~nm}$, a strong dentate peak at $203-212 \mathrm{~nm}$, and a weak dentate peak at $230-270 \mathrm{~nm}$. The infrared spectrum ${ }^{16,17}$ shows that there are skeleton vibration peaks at $6250 \mathrm{~nm}$ and $6670 \mathrm{~nm}$, and $\mathrm{C}-\mathrm{H}$ stretching vibration peaks at $3230 \mathrm{~nm}$ and $3300 \mathrm{~nm}$. Their corresponding energy can be calculated using Planck formula.

$E=\frac{h c}{\lambda}$

In (38), $E$ is the energy, $\lambda$ is the wavelength of the absorption peak, refer to (24) and (30) $c$ and $h$ and csubstitute(38). Calculation results are shown in Table 1.

Table 1 The energy corresponding to the wavelength peak of the ultraviolet and infrared spectra of benzene 


\begin{tabular}{cccc} 
Wavelength $(\mathrm{nm})$ & energy $E(\mathrm{eV})$ & Wavelength $(\mathrm{nm})$ & energy $E(\mathrm{eV})$ \\
\hline 184 & $E_{184}=6.786$ & 3230 & $E_{3230}=0.387$ \\
203 & $E_{203}=6.151$ & 3300 & $E_{3300}=0.379$ \\
212 & $E_{212}=5.890$ & 6250 & $E_{6250}=0.200$ \\
230 & $E_{230}=5.429$ & 6670 & $E_{6670}=0.187$ \\
270 & $E_{270}=4.625$ & &
\end{tabular}

Note: The energy $E$ subscript is the peak of the spectral wavelength.

In the infrared spectra ${ }^{7,17}$ of Table 1, the average value of the skeleton vibration energy representing the fundamental vibration of the benzene ring is $E_{6460}=0.192 \mathrm{eV}$. C $\mathrm{CH}$ bending vibration can be regarded as the characteristic of expansion and contraction caused by the benzene ring resonance. The average energy is $E_{3265}=0.380 \mathrm{eV}$. The - $\beta$ corresponds to the energy level difference of the molecular orbital. Combining the combination of these three basic indicators has a clear meaning, which is to simulate and quantitatively explain the results of ultraviolet spectroscopy. See Table. 2.

Table 2 Comparison of the energy corresponding to the wavelength of the benzene ultraviolet spectrum and the calculated values of some basic indicators

$U V$ Spectral measurement value Basic index calculated value (eV) Error rate (\%)

$\lambda(\mathrm{nm}) \quad E(\mathrm{eV})$

184

6.786

$-8 B+E_{3265}=6.873$

1.282

203

6.151

$-8 B-E_{3265}=6.113$

0.618

212

5.890

$-7 B+E_{6460}=5.876$

0.238

231

5.429

$-7 B-E_{6460}=5.492$

1.604

270

4.625

$-6 B-E_{6460}=4.680$

1.189 
The analysis results from Table 1, Table 2 and (30) show that the energy corresponding to the wavelength of the ultraviolet spectrum of benzene is within the range of the ground state transition energy. The combined energy value of three simple indicators shows that the ultraviolet spectrum of benzene is related to whether the benzene molecule absorbs part of the energy of the external system before the ground state transition, and the amount of ultraviolet light energy absorbed during the transition.

It is found that the ratio of enertgy $5.890 \mathrm{eV}$ corresponding to the median wavelength of $212 \mathrm{~nm}$ to the ground state transition energy of $6.493 \mathrm{eV}$ is 0.907 . The weird thing is that the ratio of the benzene ring bond length $0.1397 \mathrm{~nm}$ to the single bond length $0.154 \mathrm{~nm}$ of ordinary chain hydrocarbons is same.

It is found that the ratio of enertgy $5.890 \mathrm{eV}$ corresponding to the median wavelength of $212 \mathrm{~nm}$ to the ground state transition energy of $6.493 \mathrm{eV}$ is 0.907 . The weird thing is that the ratio of $0.1397 \mathrm{~nm}$ of the benzene ring bond length to $0.154 \mathrm{~nm}$ of the ordinary single bond length in chain hydrocarbons is also 0.907 .

$$
\frac{5.890}{6.493}=\frac{0.1397}{0.1540}=0.907
$$

39

In (39), if 6.493-5.890 $=0.603 \mathrm{eV}$, the difference between these two energies can be regarded as the holding energy of the benzene ring bond length of $0.1397 \mathrm{~nm}$. It also ensures the normality of the planar structure of the benzene molecule.The median wavelength peak at $212 \mathrm{~nm}$ may be closely related to the bond length of the benzene ring. So, quantitative relationship of median peak wavelength in UVspectrum of benzene. Or there may be a quantitative relationship between the bond length of the benzene ring and the average wavelength of the ultraviolet spectrum.

\section{3)Measuring the bond length of the benzene ring with the median peak wavelength in the ultraviolet spectrum of benzene}

The physical concept of the wave function in quantum mechanics can be understood as follows: it must meet the three so-called reasonable conditions of continuous, single value and finite in all variable regions of variable $x$. Hence, the equation ${ }^{13}$

$$
\frac{d \psi}{d x}-i k \psi=0
$$

40

Solutions have to

$$
\psi=e^{i k x}=\cos k x+i \sin k x
$$


Only when $k$ is not an imaginary number or a complex number can the above three conditions be met. Reference to the equation of momentum in quantum mechanics ${ }^{20}$ is

$$
\frac{d \psi}{d x}-\frac{i 2 \Pi p}{h} \psi=0
$$

42

The wave function is

$$
\psi=e^{i 2 \Pi x p / h}
$$

43

When a free particle moves in a circle along a ring with a radius of $r$, for the wave function to satisfy the single value condition(like an equivalent electron), the wavelength $\lambda$ must satisfy ${ }^{13}$

$$
\lambda=\frac{h}{p}=\frac{2 \pi r}{q}, r=\frac{q h}{2 \pi p}, q=1,2
$$

44

In equation (44), $q$ can also be regarded as the number of energy levels. Substituting equation (44) into equation (43) to obtain the corresponding wave function:

$$
\psi=e^{i q x / r}
$$

45

According to the one-dimensional Schrodinger equation ${ }^{20}$ should have

$$
-\frac{h^{2}}{8 \Pi^{2} m} \frac{\partial^{2}}{\partial x^{2}} e^{i x q / r}=E e^{i x q / r}
$$

46

Solve the energy as

$$
E=\frac{q^{2} h^{2}}{8 \Pi^{2} m r^{2}}
$$

47

In (47), when this free particle is given two states (such as ground state and excited state of the equivalent electron) and absorbs ultraviolet light energy, the transition energy between the two energy levels $(q=1,2)$ and the absorption peak wavelength related.

$\Delta E=\frac{h c}{\lambda}=\frac{\left(2^{2}-1\right) h^{2}}{8 \Pi^{2} m r^{2}}=\frac{3 h^{2}}{8 \Pi^{2} m r^{2}}$ 


$$
\lambda=\frac{8 \Pi^{2} c m r^{2}}{3 h}
$$

48

Reference(12),(24), (29),(30), (48), Substituting the constant values of $h, m$, and $c$ into (48), the relationship between the wavelength of the absorbed light and the radius is obtained:

$$
\lambda=\frac{8 \Pi^{2} c m r^{2}}{3 h}=10.847 \times 10^{12} r^{2}
$$

49

Substituting the median of the important peak wavelength of the ultraviolet spectrum of benzene at 212 $\mathrm{nm}$, that is, $\lambda=2.12 \times 10^{-7} \mathrm{~m}$, into (49), obtains

$$
\begin{aligned}
& r^{2}=\frac{2.12 \times 10^{-7}}{1.0847 \times 10^{13}}=1.954 \times 10^{-20} \mathrm{~m}^{2} \\
& \quad r=1.398 \times 10^{-10} \mathrm{~m}=0.1398(\mathrm{~nm})
\end{aligned}
$$

50

In (50), $r$ is the equivalent electron radius calculated using the median wavelength of the ultraviolet spectrum of benzene. It is almost equal to $0.1397 \mathrm{~nm}$ of the benzene molecular bond length measured by Raman spectroscopy. It shows that the result of two kinds of spectral measurements are consistent.

\section{Design and application of new structure of benzene molecule}

In the design of the planar structural form of the benzene molecule, the dotted line represents the halfvalent $\pi$ bond. The simple form of the ground state is a double ring of a regular hexagon. The outer circle is a solid line, and the inner circle is a dashed line. The outer ring of the excited state is the same as the ground state, and the inner ring is composed of two dashed equilateral triangles that are inverted from each other. The positive triangle represents the positive location phase, and the inverted triangle represents the negative phase (see Fig. 1).

Before the chemical reaction, the benzene in the ground state must absorb energy and be activated to the excited state.(see Fig. 2)

\section{1) Substitution reactions of benzene}

According to the new theory of benzene molecular structure, the half-valent $\pi$ bonds are formed between interval carbon atoms of the benzene ring. Its cross-covered area of the electron cloud has high electronegativity. The positive ions in the reaction solution tend to the dense area of the electron cloud, and form an instantaneous positive ion bridge and interrupts the other two bonds in the triangular bond ring. The electronegativity of the hydrogen connected to the carbon at both ends of the bridge is weakened, and is easy substituted. When the hydrogen at one end is replaced first, and the positive ion of 
the bridge breaks away immediately. The triangle bond ring is restored and completes the substitution reaction. Because the position where the substitution reaction occurs is arbitrary, there are no isomers in the substituted products. ( see Fig. 3)The proces is

The multi-substitution reaction is affected by the position of the first substituent. The electronwithdrawing positioning group with high electronegativity determines that the multi-substitution reaction mostly occurs in the meta position. The electron-repellent positioning group with low electronegativity determines that the multi-substitution reaction mostly occurs in the ortho-para position.(see Fig. 4)Such as

\section{2) Addition reaction of benzene}

The new structure theory believes that benzene molecules do not contain double bonds and should not undergo addition reactions. But in fact the hydrogenation reaction and chlorination reaction of benzene are both addition reactions. The process of benzene to cyclohexane needs to be heated to $300{ }^{\circ} \mathrm{C}$ under nickel catalysis. The activation energy is high and the time is long. In this process, when the absorption energy of benzene exceeds its so-called delocalization energy of $36 \mathrm{kcal} / \mathrm{mol}$, cyclohexatriene with addition reaction conditions is generated, and the addition reaction proceeds immediately to generate cyclohexane. Cyclohexatriene appeared only as a temporary intermediate. This process can be represented by the following reaction formula.(see Fig. 5)

\section{3) The structure design of polycyclic aromatic hydrocarbons (PAH)}

In order to verify the universality of the new structural form design method, the structural forms and chemical reaction formulas of more than 50 various benzene homologs and derivatives were designed $2,7,18,19$.

Although the planar structure of PAH is complex and changeable ${ }^{17,18}$. The design only needs to be based on the classic solid-line frame of hexagonal fused rings and follow the concepts and laws of valence bond theory (especially carbon four valence). Then it is not difficult to arrange half-valent bonds between the spacer carbon atoms with dashed lines. Because it is also regular. The specific method should be noted as follows:

A. First use equal hexagonal solid lines to splice the basic frame of the molecukar.

B. Starting from the outer periphery of the molecule, add dotted lines of the half-valent bond between interval carbon atoms of the benzene ring. Under the condition of guaranteeing the four valence of carbon, try beyond the benzene ring to connect the dashed line into several large polygonal ring until must dashed lines are connected completely.

C. The big dashed polygonal ring is divided into two phases, plus and minus. The dashed circle of the negative phase is interrupted, indicating that the part is covered by the positive phase. 
D. In the last remaining central part that can no longer be linked by a dotted line, and the part that still needs to be connected, have to use a solid line to form a double bond to finish.

The design examples of the basic planar structural forms of benzene fused ring aromatic hydrocarbons. (see Fig. 6)

The design examples of carcinogenic PAH based on Pullman's theory

French chemist Pullman ${ }^{19}$ studied 35 kinds of PAH. Summarized the theory of PAH carcinogenic molecular structure. In new structural forms of PAH for 10 carcinogenic effects, all 3 highly carcinogenic PAHs were found to contain rare double bonds. (see Fig. 7)

The variety and complexity ${ }^{18}$ of benzene homologues and derivatives test the versatility and practicability of the new design method. However, the design process shows that the structural formula can not only be easily designed, but also can be used to record chemical reaction formulas.

\section{4) Design examples of Phenylbenzene structural forms and reaction formulas}

Phenylbenzene ${ }^{2,3}$ is a special molecular structure composed entirely of a benzene ring. It is not like the fused ring compound of benzene tightly joined together but the structural forms connected to each other through $\sigma$ bond.(see Fig. 8)

In Fig. 8, Hexaphenylbenzene is a very special phenylbenzene compound. It is difficult to obtain. Only under strict conditions can this reaction be realized, and the desired result can be obtained.

\section{5) Design examples of structural forms of homologue and derivative of benzene}

(see Fig. 9)

\section{6) Design examples of Chemical reaction formulas of homolog and derivative of benzene.}

Although the chemical reaction formulas of homologues and derivatives are also complicated, the structure design is changing based on chemical reaction, and there are regularities, so the design is not difficult. (see Fig. 10).

3 Three-layer benzene tube and the thickness of graphene 
If benzene can be synthesized into a three-layer benzene tube, its total thickness is easy to calculate. The three layers of benzene are connected by the length of $2 \sigma$ bonds. The top and bottom $p_{z}$ orbital are in the same direction, only adding one $p_{z}$ orbital to the length. That is $0.5 \sigma$ bond length. The total thickness should be 2.5 times the $\sigma$ bond length. (see Fig. 11)

Borrowing the bond length ${ }^{20,21}$ of the benzene ring $0.1397 \mathrm{~nm}$ represents the $\sigma$ bond length. Its 2.5 times is $0.349 \mathrm{~nm}$. This is the total thickness of the three-layer benzene tube. The thickness of graphene $\mathrm{e}^{2,20}$ is $0.334-0.335 \mathrm{~nm}$. Therefore, its total thickness is close to graphene. According to the crystal structure constant $2.46 \mathrm{~nm}$ of graphene, calculated $\sigma$ bond length is $1.420 \mathrm{~nm}$. Its 2.5 times is $3.55 \mathrm{~nm}$. They are also very close to the thickness of graphene.

What is the energy required to synthesize an $n$-layer benzene tube? Except for the top and bottom half of the benzene ring structure, the remaining $n-1$ layers are all cyclohexane-type single-ring laminated tubes. $3(n-1)$ times the energy of $C-C$ bond synthesis must be obtained from outside the system. Based on the general $C$ - $C$ bond energy ${ }^{11}$ of $3.590 \mathrm{eV}(346 \mathrm{~kJ} / \mathrm{mol})$, minus the hydrogenation heat released, that is $(n-$ 1) $B_{H}$. The total energy required is

$E_{N}=\left(3 E_{C-C}-B_{H}\right)(n-1)=(3.590 \times 3-2.165)(n-1)$

$=8.605(n-1) e V(51)$

In (51), when $n=2$, the total energy of the $3 C$ - $C \sigma$ bonds between the two layers is only $8.605 \mathrm{eV}$, which is much weaker than the total energy of $6 \sigma$ bonds in each ring. Therefore, the interlayer structural energy is much smaller than the structural energy of the layer itself.

\section{Result}

1 Using the equivalent electron-like hydrogen atom simplified model and its wave equation, it is calculated that the ground state energy level is about $-8.665 \mathrm{eV}$, and the excited state energy level is about $-2.165 \mathrm{eV}$. The ground state transition energy is about $6.493 \mathrm{eV}$. The energy corresponding to the peak wavelength of the ultraviolet spectrum of benzene is between 4.625-6.876 eV, which is within the energy range of the equivalent electronic ground state transition. The excited state transition energy is $2.165 \mathrm{eV}$, the experimental value of benzene hydrogen heat is $2.160 \mathrm{eV}$, the difference is $0.231 \%$. When neglecting this slight difference, the hydrogenation heat of benzene becomes a constant composed of basic physical constants and is proportional to the Rydberg constant.

2 Calculated from the median peak wavelength of the benzene ultraviolet spectrum at $212 \mathrm{~nm}$, the bond length of the benzene ring is $0.1398 \mathrm{~nm}$, which is almost the same as the result of the Raman spectrum.

3 The mirror images of the benzene rings overlap, and multilayer benzene tubes may be synthesized. The calculated energy required is $8.605(n-1) \mathrm{eV}$. According to the bond length of the benzene ring, the calculated thickness of the three-layer benzene tube is $0.349 \mathrm{~nm}$, or the thickness of the three-layer 
benzene tube calculated according to the graphene microcrystalline structure constant is $0.355 \mathrm{~nm}$. They are basically close in $0.334-0.335 \mathrm{~nm}$ of the thickness of graphene.

\section{Discussion}

1 Based on the nature of chemical bonds, a new concept of covalent bonds is proposed, that is, every electron shared between atomic nuclei corresponds to a half-valent bond. In this way, it breaks the traditional concept of valence bond theory that there are only integer valence bonds, and is more widely applicable to the valence bonds of all molecules, and perfects the valence bond theory. In particular, in the excited state of the benzene molecule, half-valent bonds are formed between interval carbon atoms of the benzene ring, which clarifies the true structure of benzene and completely solves the structural problem of the benzene molecule. It also effectively explains that there are no isomers of benzene mono-substitution, and multi-substitution reactions always occur in the meta or ortho-para position.

2 Under the new theory, the half-valent bond is represented by the dashed line, and the designed planar structural form of the benzene molecule is real and novel, and becomes the new symbol of the benzene molecule. Moreover, the chemical reaction can also be recorded process in detail. Produced to replace Kekule structure used for more than a hundred years, and eliminated the useless Robinson structure, so that the benzene molecule has its own structure. With new design methods, it is easy to design the complex structural formulas and chemical reaction formulas of benzene and its homologues and derivatives, which have a wide range of applicability and lay the foundation for popularization and application.

3 The new structural formula, which can be used as the expression of the substitution reaction mechanism through the positive ion bridge effect, can be perfectly explained at the same time, the electrophilic substitution and nucleophilic reaction of benzene, and the multi-substitution reaction mechanism. This will also play a guiding role in the directional synthesis of multiple substitutions of benzene.

4 Regarding the mechanism of benzene addition reaction, the new theory is that benzene molecules do not have double bonds and cannot undergo addition reactions. Only through high activation energy, benzene turns into a cyclohexatriene intermediate, generates three double bonds, and instantaneous addition reaction occurs to generate monocyclic hydrocarbons or chain hydrocarbons. The intermediate cyclohexatriene is unstable. Therefore, it is wrong to use cyclohexatriene as the molecular structure of benzene.

5 In order to avoid the unsolvable problem of the multi-electron Schrodinger equation of benzene, the simplification of the equivalent electron hydrogen-like atom model is used to establish the wave function and wave equation, and solve the equation to obtain the ground state and excited state energy levels and transition energies. In the end, the calculated value of quantum mechanics is highly consistent with the experimental results. It not only verified the reliability of the equivalent electronic model and the new 
structural theory, but also quantitatively explained the unsolved problems such as the hydrogenation heat of benzene and ultraviolet spectroscopy.

6 Using the median peak wavelength of the UV spectrum of benzene, the calculated bond length of the benzene ring is almost identical to the measured value of the Raman spectrum, indicating the consistency of the measurement results of the two spectra.

7 Based on the new structure theory of benzene molecules, the benzene rings are stacked in mirror images, and it is possible to synthesize multilayer benzene tubes. Calculate the thickness of the threelayer benzene tube, which is equivalent to the thickness of graphene. When considering that graphene also exhibits a high degree of chemical stability, good electrical and thermal conductivity, as well as high hardness and toughness, it is much like metal 22 with a complex crystal lattice. Therefore, graphene is more like a three-dimensional structure. It is speculated that there is at least one type of graphene with a three-layer structure. In this three-layer structure, the centerline of the carbon tube formed by a large number of equilateral hexagonal carbon rings may be an undisturbed superconducting channel. It is an ideal superconducting structural material in itself.

\section{Declarations}

Disclaimer:

This research process has nothing to do with all institutions. For all authors, there is no academic or economic competition.

\section{References}

1 Kauffman, G. B. Essays on the history of organic chemistry (Traynham, James G.) Journal of Chemical Education 65, 7, A188 (1988).

2 Wade, L. G. Wade. Organic Chemistry. (Prentice Hall, 2009).

3 Carey, F. A. \& Sundberg, R. J. Advanced Organic Chemistry: Structure and Mechanisms Part A. (Springer, 2008).

4 Katsnelson, M. I. Graphene. (Cambridge University Press $₫ 2012)$.

5 Klein, D. J. \& Trinajstic N. Valence Bond Theory and Chemical Structure. (Elsevier, 1990).

6 Levine, I. N. Quantum Chemistry. (Pearson Education, 2013).

7 Rae, A. I. M. \& Napolitano, J. Quantum Mechanic. (CRC Press, 2015).

8 Lowe, J. P. \& Peterson, K. Quantum Chemistry. (Academic Press, 2005). 
9 Pauling, L. The Modern Theory of Valence. Journal of the Chemical Society 76, 1461-1467 (1948).

10 Bent, H. A. Molecules and The Chemical Bond: An Introduction to Conceptual Valence Bond Theory. (Trafford Publishing, 2013).

11 Sanderson, R.T. Chemical Bond and Bond Energy. (Academic Press, 1977).

12 Corry, S. Symmetry and Quantum Mechanics. (Chapman and Hall/CRC, 2016).

13 Zeidler, E. Nonlinear Functional Analysis and its Application III: Variational Methods and Optimization. (Springer, 1985).

14 Simon, B. Quantum Mechanics for Hamiltonians Defined as Quadratic Forms. (Princeton University Press, 2016).

15 Putz, M. V., Cimpoesu, F. \& Ferbinteanu, M. Structural Chemistry. (Springer International Publishing, 2017)

16 Huber, K.P. and Herzberg, G. Molecular Spectra Molecular Structure IV. Constants of Diatomic Molecules. (Van Nostrand Reinhold Company, New York, 1979).

17. Chandra, S. Molecular Spectroscopy. (Alpha Science International Ltd, 2009).

18 Hoard, J. L., Geller, S. \& Cashin, W. M. Structures of Molecular Addition Compounds. III. AmmoniumBoron Trifluoride, $\mathrm{H}_{3} \mathrm{~N}_{-} \mathrm{BF}_{3}$. Acta Crystallographica 4, 396-398 (1951).

19 Pullman, A. et al: Adu. Cancer Reseach, Vol 3 Acadmic Press (1955), 117

20 Novoselov, K. S., Geim A. K., Morzov S., Jiang, D., Zhang, Y., Dubonos, S.V., Grigorieva, I.V. \& Firsov, A. A. Electric field effect in atomically thin carbon films. Science, 306(5696), 666-669 (2004).

21 Lai, D., Chen, W. \& Jiang G. Research Progress of Graphene-based Composite Materials. Current Physical Chemistry 3, 269-282 (2013).

22 Cao, Y., Fatemi, V., Fang, S., Watanabe, K., Taniguchi, T., Kaxiras, E. \& Jarillo-Herrero, P. Unconventional superconductivity in magic-angle graphene superlattices. Nature 556, 43-50 (2018).

\section{Figures}


<smiles>c1ccccc1</smiles>

Ground state

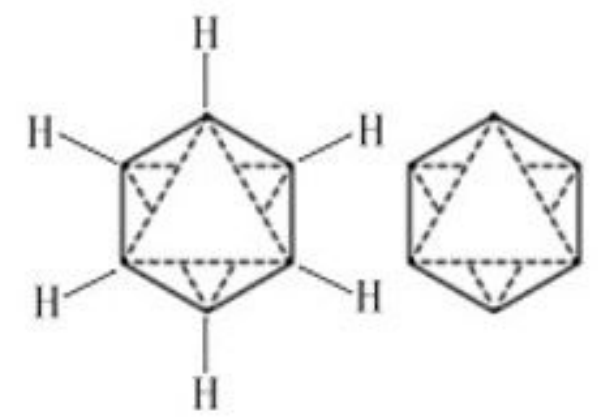

Excited state

\section{Figure 1}

New plane structural forms of benzene molecules

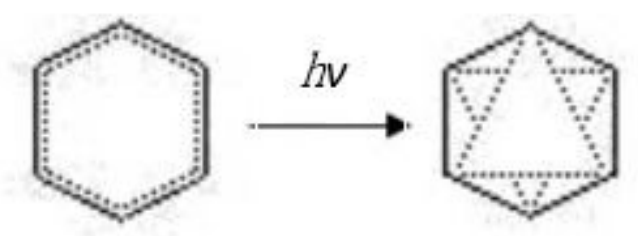

Ground state

Excited state

Figure 2

Benzene molecule ground state activated to excited state

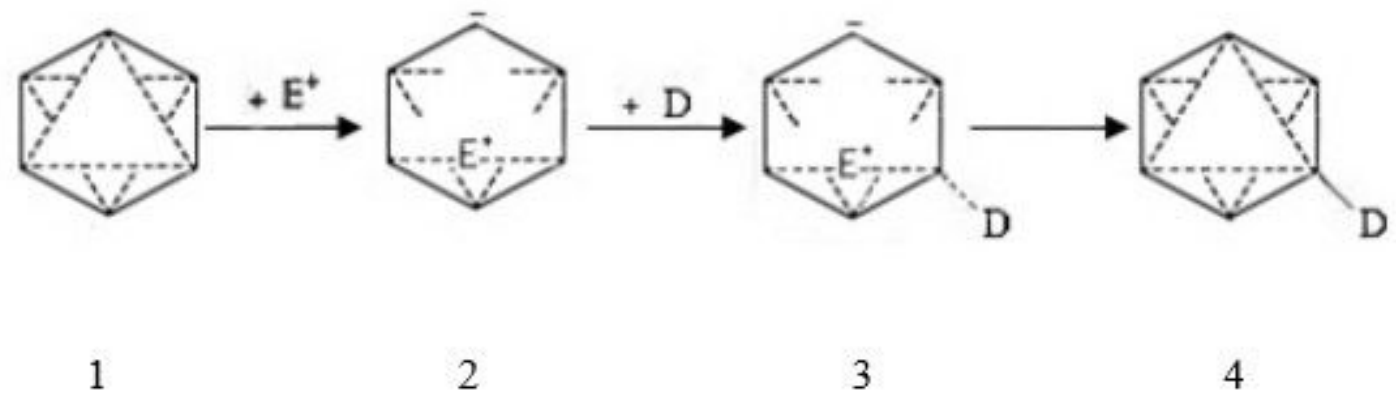

1 Excited state of benzene molecule 2 Formation of positive ion bridge 3 Monosubstituted benzene is being generated 4 Benzene ring structure recovery

Figure 3

Process of benzene mono-substitution reaction 


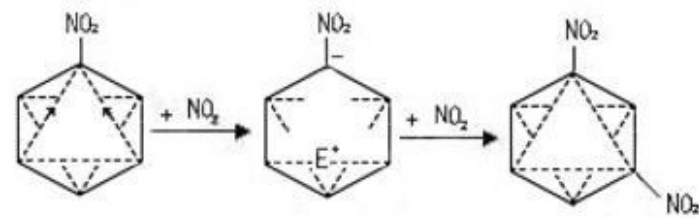

1,3-m-Dinitrobenzene substitution reaction

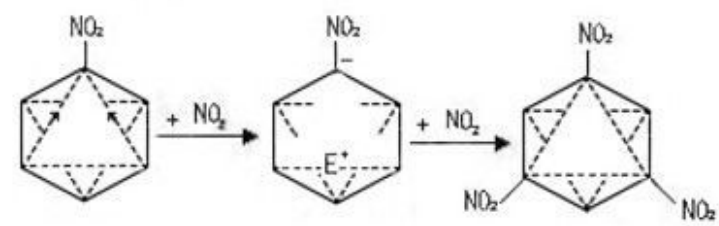

1,3,5-m-trinitrobenzene substitution reaction

Meta-substitution reaction of benzene

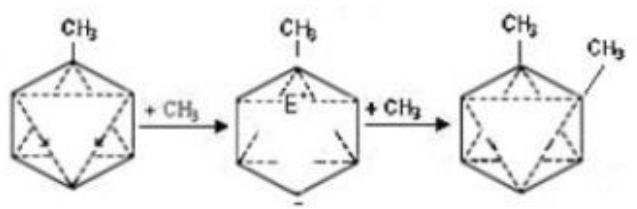

Toluene

1,2-o-xylene

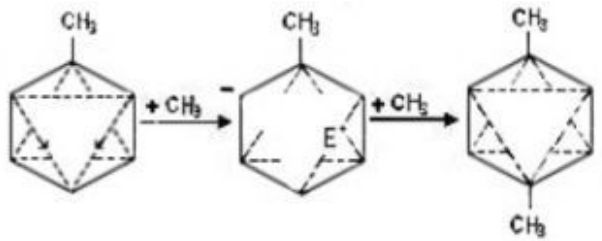

Toluene

1,4-p-xylene

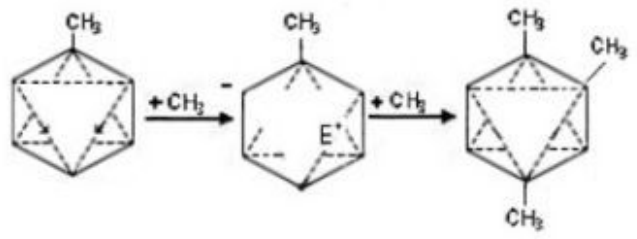

Toluene

1,2,4-ortho-para-trimethylbenzene

ortho-para position substitution reaction of benzene

Figure 4

multi-substitutio reaction of benzene 


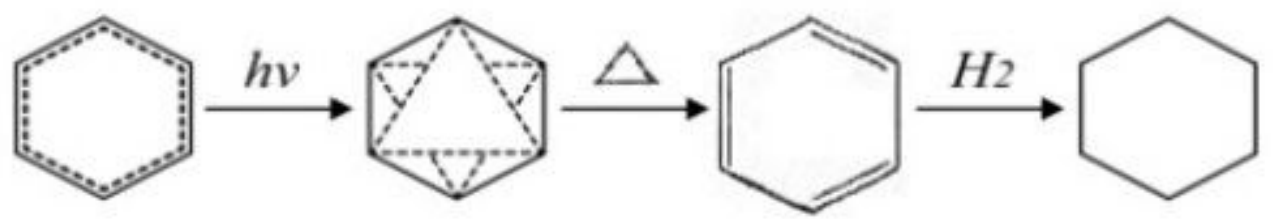

Phenyl state Phenyl excited state Cyclohexatriene Cyclohexane

\section{Figure 5}

The addition reaction of benzene to cyclohexane

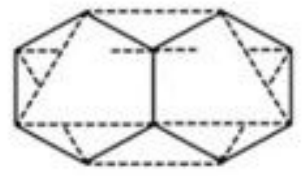

Naphthalene

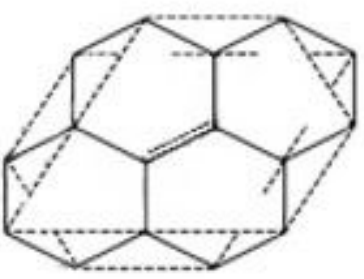

Pyrene

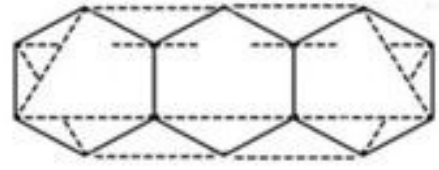

Anthracene

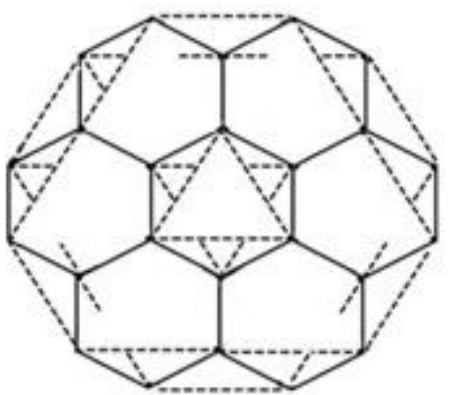

Guanidine

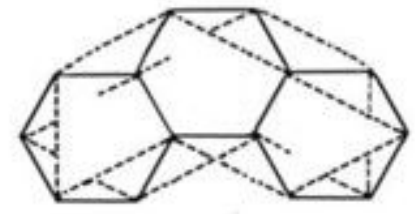

Phenanthrene

\section{Figure 6}

Basic structural forms examples of benzene fused ring aromatic hydrocarbons 


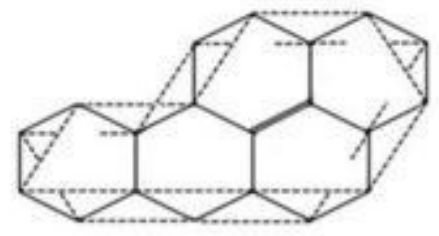

3,4-Benzopyrene

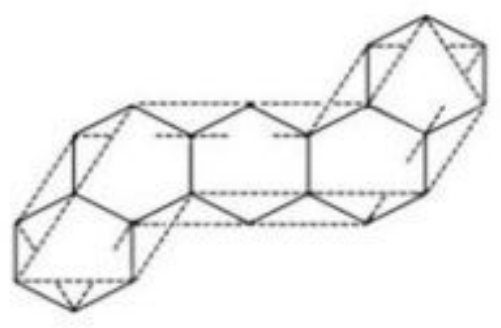

1,2,5,6-Dibenzoanthracene

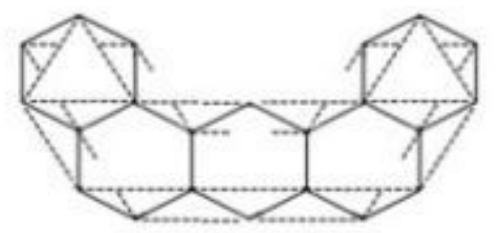

1,2,3,4-Dibenzoanthracene

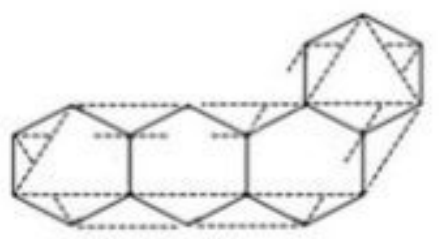

1,2-Benzoanthracene

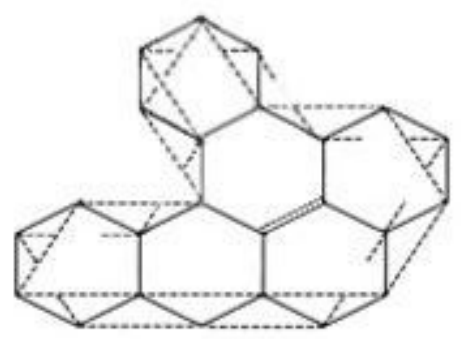

1,2,3,4-Dibenzopyrene

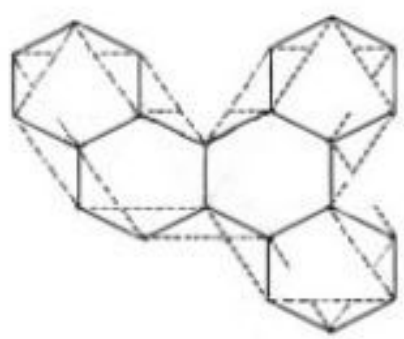

1,2,3,4-Dibenzophenanthrene

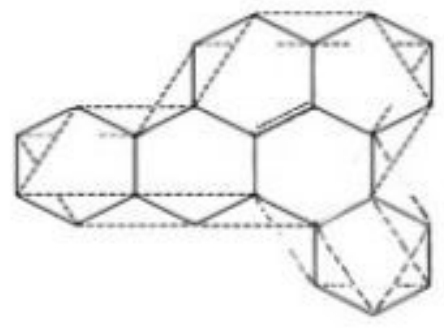

3,4,6,7-Dibenzopyrene

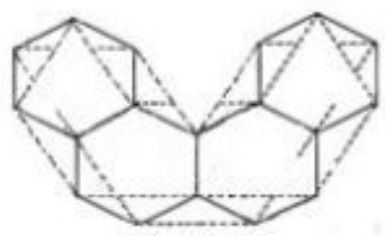

3,4-Benzophenanthrene

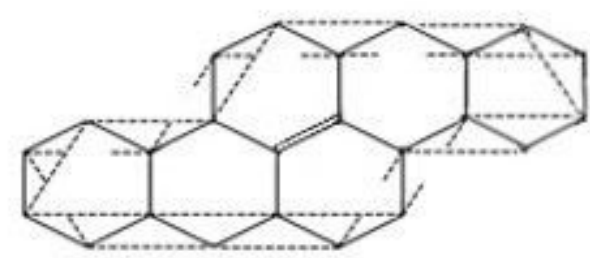

3,4,8,9-Dibenzopyrene

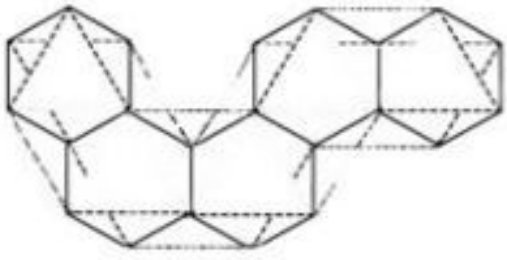

1,2,5,6-Triphenylene

\section{Figure 7}

Pullman theory 10 cases of PAH carcinogenic molecular new plane structure 


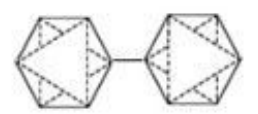

Bipheny1

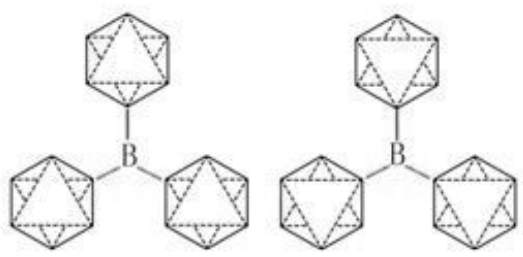

(Positive phase) (reversed-phase)

Triphenylboron

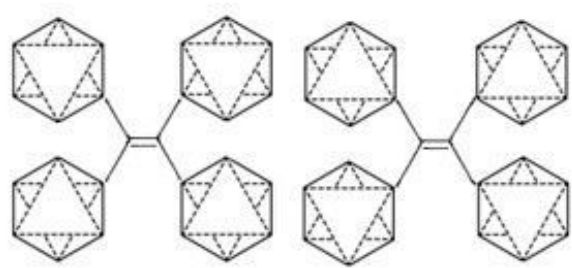

(Positive phase) (reversed-phase)

Tetraphenylethylene

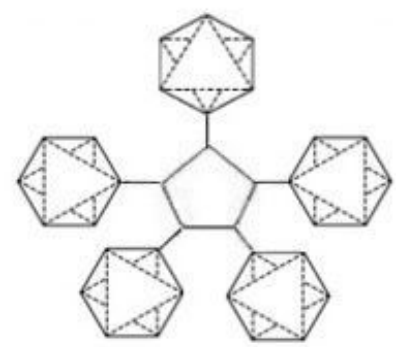

Pentaphenylcyclopentane

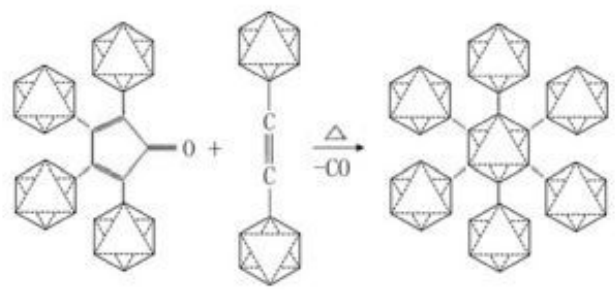

Tetraphenylcyclopentadienone Diphenylacetylene Hexaphenylbenzene

\section{Figure 8}

Cycloaddition of tetraphenylcyclopentadienone and diphenylacetylene to produce hexaphenylbenzene 


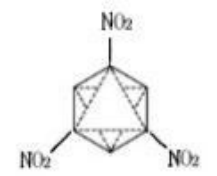

1,3,5-Trinitrobenzene

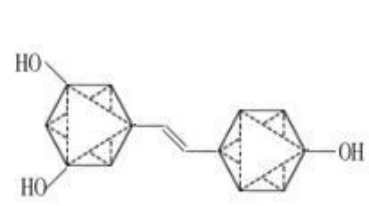

Resveratrol

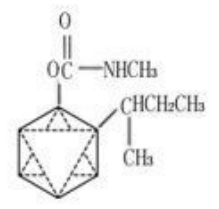

Basa<smiles>CNC(=O)OC12CC3CC(C1)C(Cl)(Cl)C3C2</smiles>

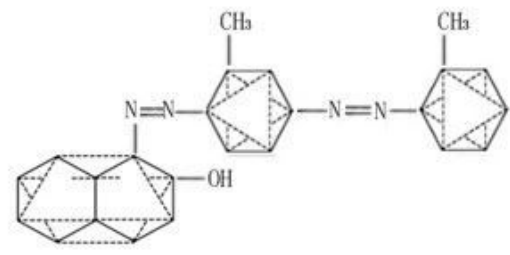<smiles>CC1C2CC3(C(=O)O)C1C2(O)C3(O)O</smiles>

Metronidazole

Sudan Red IV

Gallic Acid<smiles>CC1=C(C(=O)O)N2C(=O)C(NC(=O)C(N)C3C4C5C6C3C3C4C5C63)C2SC1</smiles>

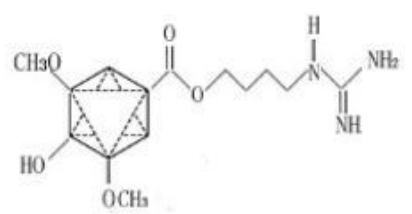

Cephalexin

Leonurusine
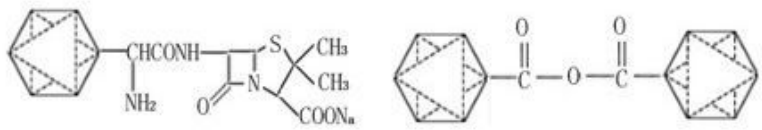

Ampicillin Sodium

Benzoic Anhydride<smiles>COC12C3C4C1C1C2C3C41C1C(O)C(O)C(C)N1C</smiles>

Codonopsis

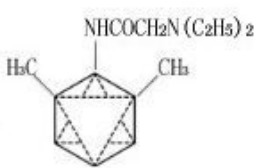

Ledocaine

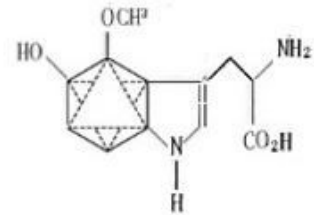

Serotonin

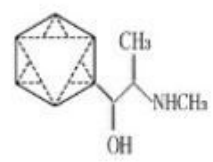

Ephedrine

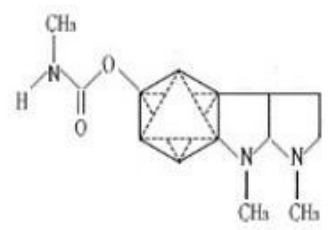

Physostigmine

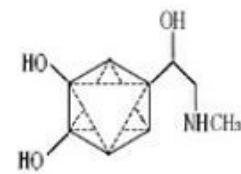

Adrenal glands

Figure 9

New structural formulas of benzene homologous derivatives 

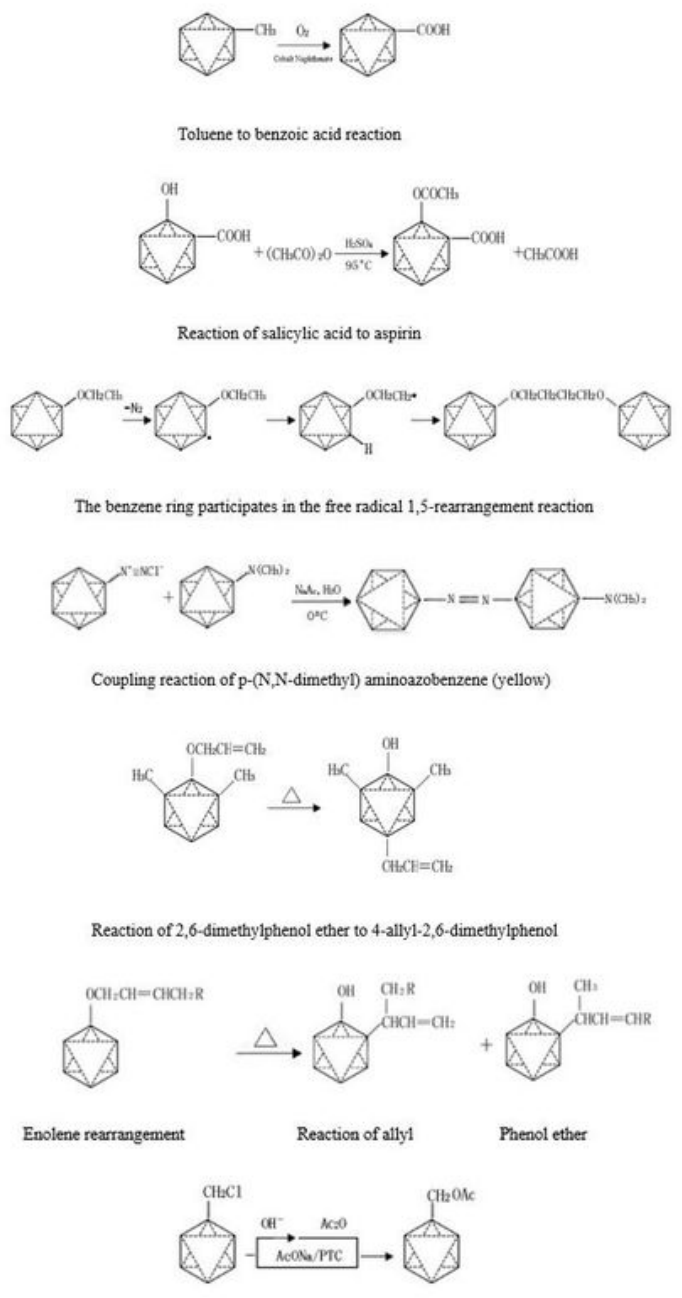

Synthesis of benzyl acetate from benzyl chloride
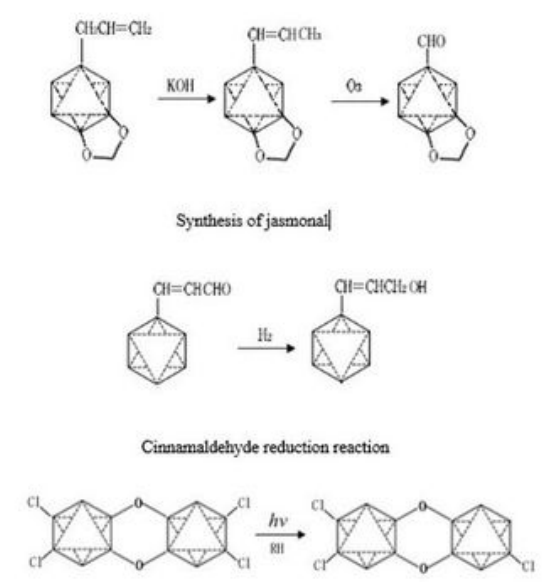

The reaction of dioxins in the hydrocarbon solution replacing chlorine with hydrogen

\section{Figure 10}

Design example of the chemical reaction formula of the benzene homolog 


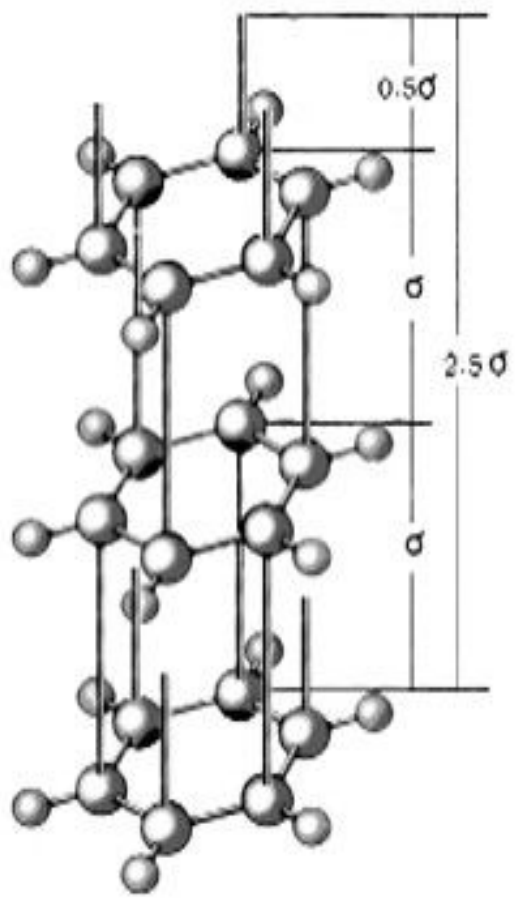

Figure 11

Thickness model of three-layers benzene tube

\section{Supplementary Files}

This is a list of supplementary files associated with this preprint. Click to download.

- economiccompetiti1.docx 